\title{
A Reliable Method for Measurement and Normalization of Pediatric Hippocampal Volumes
}

\author{
ANDRE OBENAUS, CHARLOTTE J. YONG-HING, KAREN A. TONG, AND GORDON E. SARTY
}

Department of Medical Imaging, University of Saskatchewan, Saskatoon, Saskatchewan, Canada

\begin{abstract}
A robust standardized method for segmentation, quantification, and normalization of pediatric hippocampal volumes using magnetic resonance imaging is presented. The method will find application in time course measurements of hippocampal volumes in pediatric patients who suffer from temporal lobe epilepsy and was tested prospectively on six control patients (13-60 mo of age). The un-normalized hippocampal volumes obtained using our segmentation method ranged from 3.85 to $6.38 \mathrm{~mL}$, in agreement with previously published results. Inter- and intraobserver variability of the segmentation method was determined to be $13.3 \%$ and $2.8 \%$, respectively. Four different methods of volume normalization were tested. Normalization is required to
\end{abstract}

\section{ABSTRACT}

adjust for age-related increases in hippocampal volume. The normalization approach that seemed to compensate best for growth-related hippocampal volume changes was based on a simple estimation of intracranial volumes. This is the first report of a consistent and reliable method for segmentation and normalization of hippocampi from pediatric patients that can be used to study the progression of neurologic diseases in children. (Pediatr Res 50: 124-132, 2001)
Abbreviations
MRI, magnetic resonance imaging
TLE, temporal lobe epilepsy

Noninvasive neuroimaging techniques can be used to examine the structural and pathophysiologic bases underlying numerous disease processes. MRI is the imaging technique of choice for evaluating patients with epilepsy inasmuch as TLE often leads to anatomic lesions (1).

The primary features of the mesial temporal sclerosis associated with TLE can be detected with MRI, and these features correlate well with known histologic features $(2,3)$. Hippocampal atrophy and increased signal on $\mathrm{T}_{2}$-weighted magnetic resonance (MR) images can be determined by qualitative visual analysis of MR images. Although visual analysis of hippocampal sclerosis can achieve a sensitivity of $98 \%$ and a specificity of $93 \%$, the additional advantages of quantitative volume analysis include reliable lateralization of atrophy and increased objectivity of analysis (3). Absolute hippocampal volumes measured from fast spin-echo MR images have been shown to correlate with the hippocampal neuronal density of CA1, CA2, and CA3; such a correlation was absent with visual analysis of increased $\mathrm{T}_{2}$ signal (4). Van Paesschen et al. (5) demonstrated that increased $\mathrm{T}_{2}$ signal has a neuropathological

Received September 28, 2000; accepted January 16, 2001.

Correspondence: Dr. Andre Obenaus, Department of Medical Imaging, University of Saskatchewan, 103 Hospital Dr., Saskatoon, SK S7K 0N7, Canada; e-mail: obenausa@levade.usask.ca

Supported, in part, by the University of Saskatchewan's College of Medicine, Department of Medical Imaging, and the Medical Research Council of Canada. A.O. and G.S. are Medical Research Council of Canada Research Scholars.

Current address for Dr. Tong: Department of Radiology, Loma Linda University, Rm B623, P.O. Box 2000, Loma Linda, CA 92324, U.S.A. basis different from that of hippocampal volume loss. Thus, hippocampal volumetric analysis can provide a reliable, noninvasive method for identification of hippocampal sclerosis from MRI.

Visual analysis of MR images may detect gross hippocampal atrophy, but smaller degrees of volume loss may be overlooked and small asymmetries of shape may be misinterpreted as volume loss. Both histologic and volumetric studies have shown that hippocampal sclerosis in TLE tends to occur ipsilateral to the epileptogenic temporal lobe, but bilateral volume loss can also be present $(4,6,7)$. Side-to-side comparison of hippocampal volumes can identify hippocampal sclerosis on the atrophic side, but absolute hippocampal volumes must be used to reliably detect bilateral atrophy $(1,4)$. Correcting hippocampal volumes for total intracranial volume has been shown to further increase sensitivity to bilateral hippocampal sclerosis (8).

Hippocampal volumes can be calculated using either manual, semiautomatic, or completely automatic computer segmentation of sequential MR images. The majority of hippocampal volume studies reported have used manual segmentation techniques $(6,8-14)$. Because the pediatric hippocampus is still developing, it is smaller and less well defined than the adult hippocampus, thus both automatic and manual segmentation are more difficult. No system, manual or automatic, specifically designed for outlining the pediatric hippocampus has been reported before our study. 
Our methodology for pediatric hippocampal segmentation on MRI was developed to investigate whether the hippocampal volumes of children could be reliably quantified. A flowchart was developed using a generally accepted protocol for segmenting the adult hippocampus as a starting model (13). We used the resulting schema to segment and compute the volume of the hippocampus on MR images of control pediatric patients. It is anticipated that the flowchart will be vital for the development of an automatic computer segmentation algorithm.

Because of age-related differences of hippocampal volumes in children from our study and the need to ascertain absolute volumes, four alternate methods for the normalization of pediatric hippocampal volumes were tested with the aim of finding an age-independent measure. The methods, in order of increasing complexity, normalized the volumes relative to 1) head circumference, 2) brain volume estimation using an equation for a sphere, 3) a standardized "block volume" of brain encompassing the entire hippocampus, and 4) intracranial volume estimation. The last method resulted in the best normalization for our data set of pediatric patients. Normalization will be particularly useful for the sequential follow-up of epilepsy cases.

\section{METHODS}

\section{Patient Selection and Study Subjects}

Children aged 1 mo to $16 \mathrm{y}$ who were undergoing MRI of the spine or being investigated for posterior fossa tumors were eligible as controls. All MR images were obtained under general anesthesia in accordance with established procedures at our site for pediatric patients (up to $10 \mathrm{y}$ of age). Patients were admitted between October 1, 1997, and December 31, 1998. A staff neuroradiologist (K.A.T.) selected the patients. Informed consent was obtained from the patients or their legal guardians in accordance with the approved institutional ethics review committee.

Six control patients with no history of seizures were identified over the study period, and their demographic information and diagnoses are summarized in Table 1. Their ages ranged from 14 to $60 \mathrm{mo}$, with an average age of $44.6 \mathrm{mo}$. There were four males and two females in this group. These patients presented with a variety of clinical diagnoses that did not involve the hippocampus or temporal lobe or affect brain volume. The cranial MRI studies in these patients were confirmed to be normal.

\section{MR Image Acquisition}

MRI studies were performed on a 1.5-T Siemens Magnetom-SP (Erlongen, Germany). Scout sequences were obtained to

Table 1. Summary of patients used for hippocampal volume measurements

\begin{tabular}{clccl}
\hline & & $\begin{array}{c}\text { Age at scan } \\
(\mathrm{mo})\end{array}$ & $\begin{array}{c}\text { Weight } \\
(\mathrm{kg})\end{array}$ & \multicolumn{1}{c}{ Reason for imaging } \\
\hline 1 & M & 42 & 14 & Neurofibromatosis \\
2 & M & 52 & 21 & Spinal cord tumor \\
3 & F & 55 & 20 & Spinal neuroblastoma \\
4 & M & 14 & 11 & Spinal nerve root abnormality \\
5 & M & 60 & 18 & Blue spells \\
6 & F & 31 & 13 & Scoliosis \\
\hline
\end{tabular}

insure proper positioning of the patient's head. $\mathrm{T}_{1}$-weighted images were collected with a three-dimensional (3D) MP-RAGE (magnetization prepared-rapid acquisition gradient echo) sequence. The images were obtained perpendicular to the superior margin of the right temporal lobe at the level of the middle temporal gyrus in a $20^{\circ}$ tilt from the coronal plane. The sequences had the following parameters: TI/TR/TE/flip angle $=300 \mathrm{~ms} / 12.5$ $\mathrm{ms} / 5 \mathrm{~ms} / 10^{\circ}$. The field of view was $25 \times 25 \times 64 \mathrm{~cm}$ and a 130 $\times 256 \times 64$ acquisition matrix was used. The data were Fourier transformed into $256 \times 256 \times 64$ voxel data sets. An $80-\mathrm{mm}$ slab with a total of 64 images for an effective slice thickness of 1.25 $\mathrm{mm}$ was used. Acquisition time was $4 \mathrm{~min} 48 \mathrm{~s}$.

The $\mathrm{T}_{1}$-weighted images used in this study show the cerebrospinal fluid (CSF) of the ventricles as the darkest pixels, gray matter as distinctly lighter pixels, and white matter as even lighter pixels (close to white). Blood vessels appear brightly white and were excluded from our traces and remnants of the hippocampal sulcus, appearing as dark pixels within the region of interest, were included in our measurement of hippocampal volume. When the choroid plexus was visible within the ventricle, it had a lighter more heterogeneous appearance and made delineation of the hippocampus difficult.

\section{MR Image Analysis}

The MR images were transferred to a dedicated Windows NT workstation and all image analysis was done offline using Cheshire software (Hayden Image Processing Group, Waltham, MA, U.S.A.). Manually adjusted windowing was used to ensure consistent contrast throughout the study. Images were assessed on a slice-by-slice basis to obtain optimal contrast to visualize the anatomic landmarks for segmentation. Rough regions of interest (ROIs) were initially segmented automatically using a proprietary tool that found the best-guess edge of an object. The ROI was then adjusted manually following the flowchart we developed (see "Hippocampal Segmentation" below). The total time required for a trained individual to segment and calculate the hippocampal volume was on average $1 \mathrm{~h}$. Untrained individuals required $2-3 \mathrm{~h}$ to complete the same segmentation and calculations.

From these segmented areas, the volumes, in cubic centimeters, were automatically calculated and rendered. Tracings of the hippocampus were based on anatomical landmarks previously defined by others $(13,15)$. The staff neuroradiologist (K.A.T.) verified all the traces for accuracy.

\section{Hippocampal Segmentation}

The first step in defining the boundaries of the hippocampus from MRI was to identify the range of MR sections that contained the hippocampus. The MP-RAGE sequence had 64 slices, of which 30 slices on average contained the pediatric hippocampus. After the full range of MR image sections containing the hippocampus were identified, segmentation could begin (Fig. 1).

The hippocampus forms an arc and has three primary segments from anterior to posterior: a transversely oriented head with dilations and elevations called hippocampal digitations, a sagittally oriented body, and a tapering, transversely oriented 
tail. The hippocampus is composed of two interlocking Ushaped laminae, Ammon's horn and the dentate gyrus (16-19).

In general, the most important anatomical landmarks defining the lateral, inferior, and medial boundaries were similar for the head, body, and tail of the hippocampus. However, the superior boundary of the hippocampus differed from anterior to posterior because of changes in the surrounding anatomy. Our measurement of the hippocampal body and tail included the subicular complex, the hippocampus proper, the dentate gyrus, the alveus, and the hippocampal fimbria. We excluded the amygdala, parahippocampal gyrus, the isthmus of the cingulate gyrus, and the crus of the fornix.

\section{The Hippocampal Head}

Identification of the first MR image slice containing a portion of the hippocampus required that the pes, or head, of the hippocampus was visible and comprised the anterior boundary (Figs. 1, 2A, and 3, $A$ and $B$ ). The hippocampal head (or pes hippocampus) had a distinctive shape with a wavy superior portion that was clearly visualized in most cases. The superior boundary of the hippocampus was defined when the amygdala could be distinguished from the hippocampus in the anteriormost slices (Figs. 1, 2A, and 3, $A$ and $B$ ). The hippocampus was

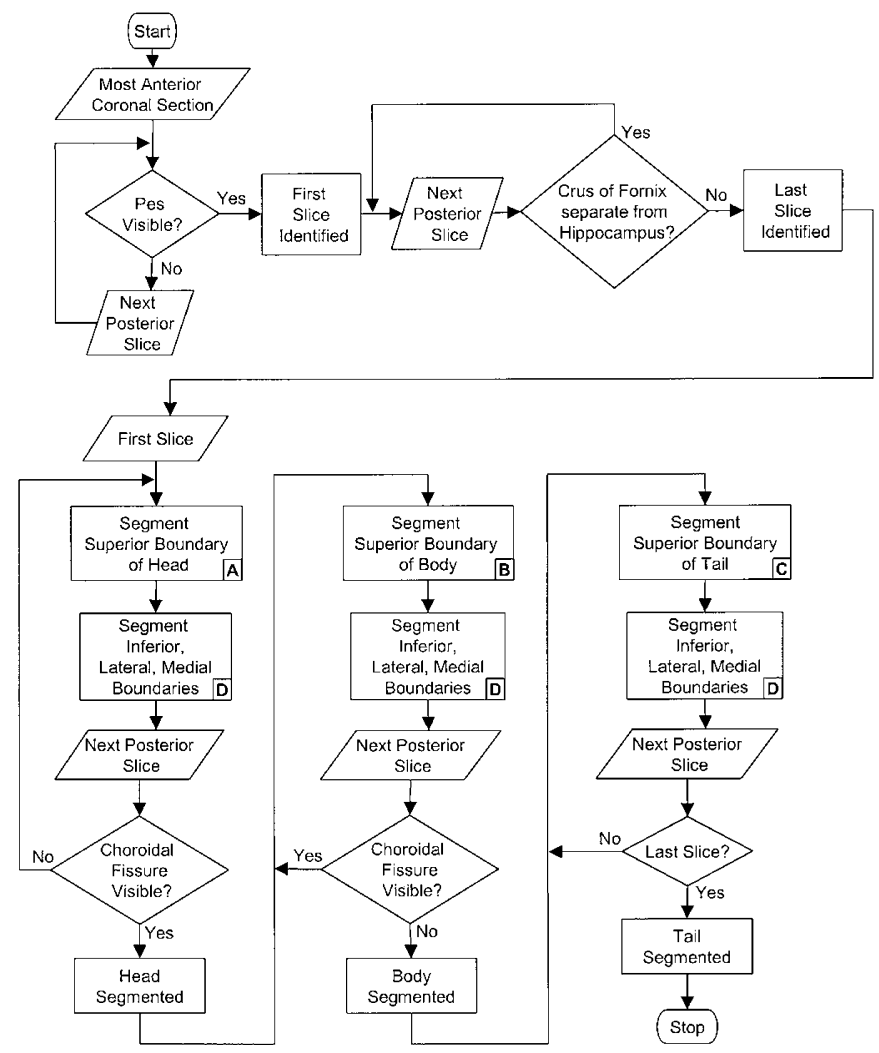

Figure 1. Schema for segmenting the pediatric hippocampus. This decision tree for segmenting the pediatric hippocampus was developed to standardize the key steps in calculating hippocampal volumes. The specific anatomical landmarks that describe the superior boundary are different for the hippocampal head, body, and tail, and the algorithms for segmenting these borders are shown in Figure 2, $A, B$, and $C$, respectively. The inferior, lateral, and medial boundaries are common throughout the length of the hippocampus, and the algorithm is detailed in Figure $2 D$. clearly separated from the amygdala on sections containing the uncal recess of the inferior horn of the lateral ventricle. The white matter of the alveus overlying the ventricular surface of the hippocampal digitations could be used as the superior boundary even when the uncal recess was not visible. The amygdala appears as a homogeneous gray mass overlying a discontinuous layer of darker pixels representing the CSF within the uncal recess.

Isolation of the hippocampus was much more difficult in those sections where the hippocampus and amygdala were fused without a visible intervening alveus or ventricle (Fig. 3, $C$ and $D$ ). When neither the alveus nor the lateral ventricles were visible, a straight horizontal line was used to demarcate the superior margin of the hippocampus from the overlying amygdala. The line was drawn from the inferior horn of the lateral ventricle to the surface of the uncus. If the semilunar gyrus was visible on the uncus, the line was drawn specifically to the sulcus at the inferior margin of the semilunar gyrus and, in this case, the line was not always exactly horizontal.

The inferior boundary of the pediatric hippocampal head extended to (but did not include) the white matter of the
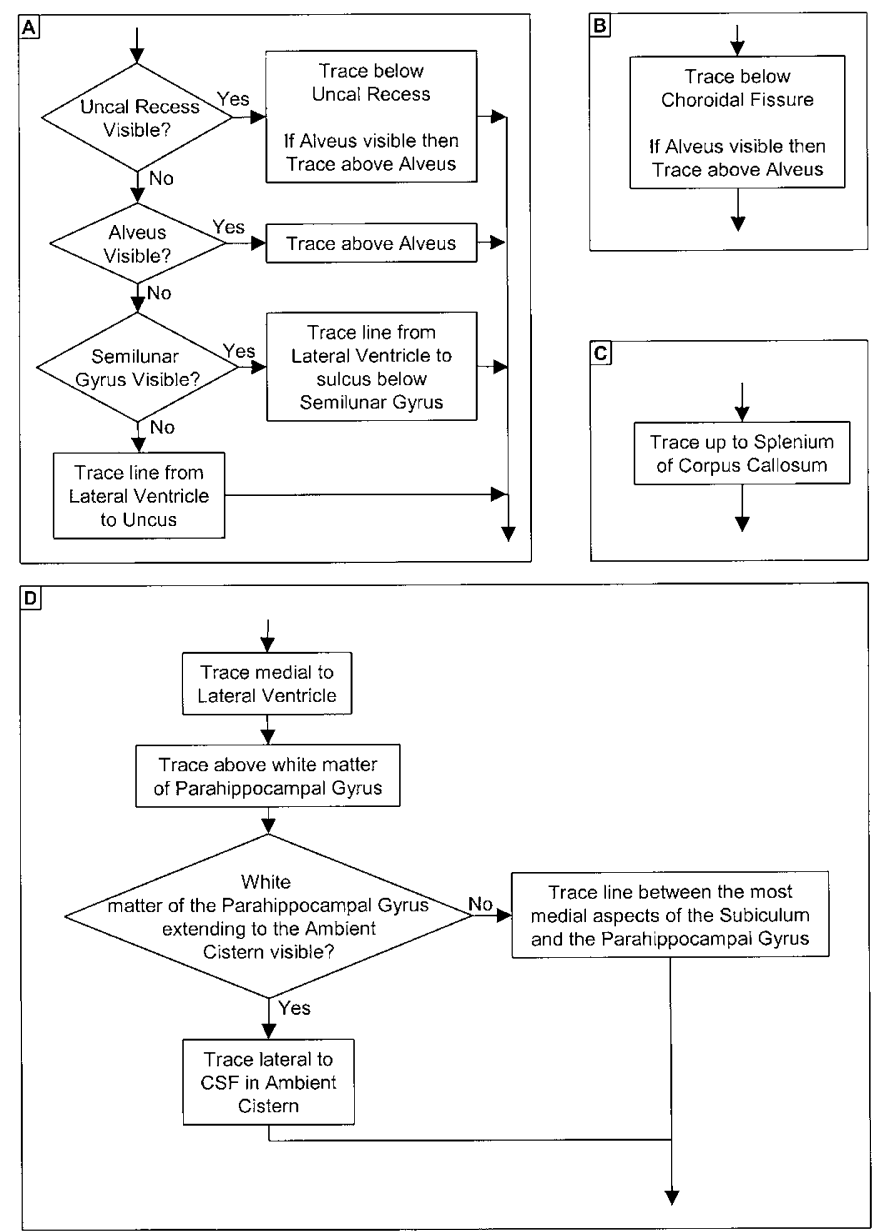

Figure 2. Specific algorithms for segmentation of the pediatric hippocampus. (A) Algorithm for segmentation of the superior boundary of the hippocampal head. $(B)$ Algorithm for segmentation of the superior boundary of the hippocampal body. $(C)$ Algorithm for segmentation of the superior boundary of the hippocampal tail. $(D)$ Algorithm for segmentation of the inferior, lateral, and medial boundaries of the hippocampus. This last algorithm is common to the head, body, and tail of the pediatric hippocampus. 

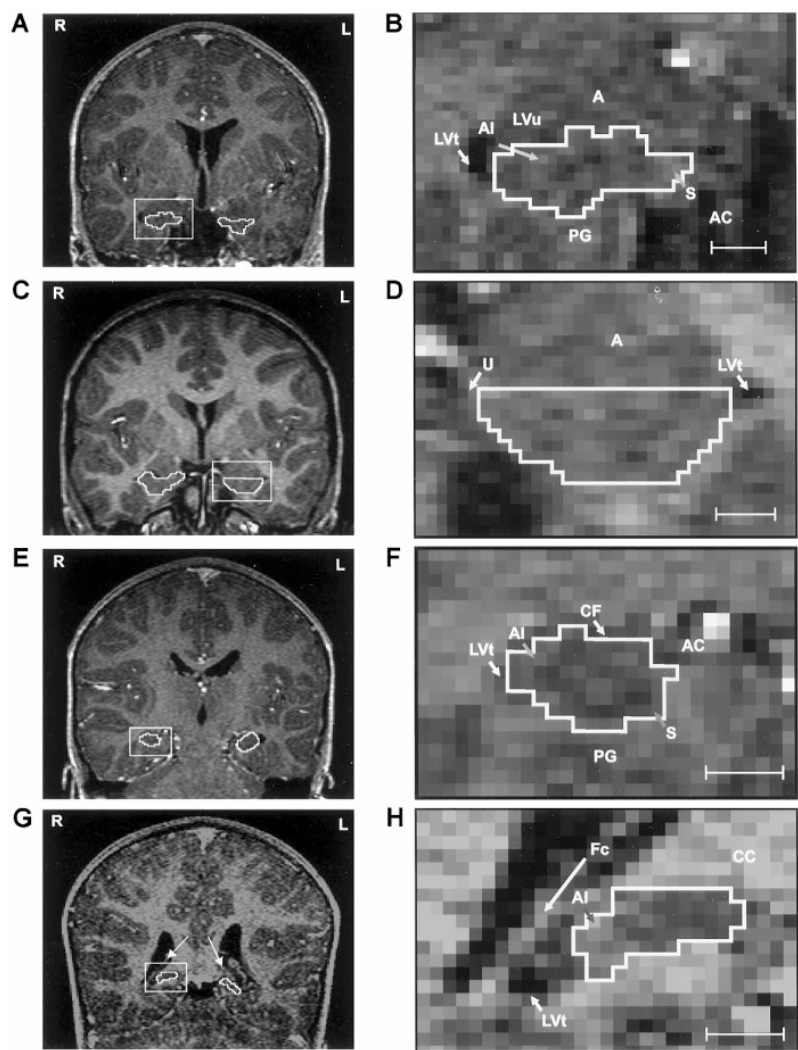

Figure 3. Segmentation of the hippocampus. A typical MRI series acquired from a 3D MP-RAGE sequence. These $\mathrm{T}_{1}$-weighted coronal images are from a 55 -mo-old pediatric patient. Calibration bar $=5 \mathrm{~mm}$. $(A)$ The hippocampal head is outlined on both sides of the image. CSF in the ambient cistern is represented by the dark gray or black pixels. The right hippocampus (within rectangle) is enlarged (see $B$ ) to illustrate the anatomical boundaries. (B) The amygdala $(A)$ is visible on both sides, overlying the hippocampus. The superior margin of the hippocampus includes the white matter of the alveus $(A l)$ and excludes the dark pixels representing the uncal recess of the lateral ventricle $(L V u)$. Laterally, the hippocampal head is traced separately from the dark pixels of the temporal horn of the lateral ventricle $(L V t)$. The hippocampus is traced out medially to the dark pixels of the ambient cistern $(A C)$. The medial boundary included the subiculum $(S)$. Inferiorly, the white matter of the parahippocampal gyrus $(P G)$ is completely excluded. The margin between the parahippocampal gyrus and the subiculum is the angle between their most medial aspects. $(C)$ When the hippocampal head is fused to the amygdala and neither the alveus nor the uncal recess of the temporal horn is visible (left side), the superior margin of the hippocampal head cannot be visualized. The alveus and uncal recess are visible on the opposite side where the superior margin can be visualized and traced. $(D)$ When the superior margin of the hippocampal head is not visible, the margin between the hippocampus and the overlying amygdala was estimated by drawing a straight horizontal line from the lateral ventricle $(L V t)$ to the surface of the uncus $(U)$. $(E)$ The hippocampal body is clearly visible on both sides of this oblique coronal MR image. The rectangle encloses area of the hippocampal body and adjacent structures enlarged in $F$. $(F)$ The superior boundary of the hippocampal body is the choroidal fissure $(C F)$, which is visible between the temporal horn of the lateral ventricle $(L V t)$ and the ambient cistern $(A C)$. The white matter of both the alveus $(A l)$ and the hippocampal fimbria (not visible in this section) are included whereas the choroid within the ventricles is excluded. The margin between the subiculum $(S)$ and the parahippocampal gyrus $(P G)$ is again traced as the angle between their most medial aspects. $(G)$ An MRI at the level of the hippocampal tail, the most posterior section from which measurements of the hippocampus are taken. The area enclosed within the rectangle is enlarged in $H$ and illustrates the crus of the fornix $\left(F_{C}\right)$ in full profile and completely separate from the hippocampus and its fimbria. $(H)$ The tail of the hippocampus extends superiorly and medially up to the splenium of the corpus callosum $(C C)$. The lateral boundary is formed by the dark pixels of the temporal horn of the lateral ventricle $(L V t)$ and the white pixels of the alveus $(A l)$. The alveus is included in our measurements of hippocampal volume. parahippocampal gyrus (Fig. 2D). The darker pixels representing portions of the lateral ventricle that extended under the inferior surface of the hippocampus were visible in some sections and were not included (Fig. 3, $A$ and $B$ ). In all sections, the gray matter of the parahippocampal gyrus (the entorhinal cortex) was excluded but the subiculum was included.

The CSF in the lateral ventricle was used as the lateral boundary, and the region traced included the alveus lining the ventricular surface of the hippocampus (Figs. $2 D$ and 3 ).

The CSF in the ambient and uncal cisterns formed the medial boundary (Figs. $2 D$ and 3, $A$ and $B$ ). On all sections, the boundary between the subicular complex and the parahippocampal gyrus was defined by the angle formed by the most medial extents of these structures. The margin between the gray matter of the subiculum and the gray matter of the parahippocampal gyrus followed the division formed by the white matter of the parahippocampal gyrus in some sections. Otherwise, the margin was traced from the most medial edge of the white matter to the most medial pixels of the subiculum. The hippocampal sulcus was also included in the measurement of the hippocampal head.

\section{The Hippocampal Body}

Visualization of the choroidal fissure indicated the slice was taken through the hippocampal body (Figs. 1, 2B, and 3,E and $F)$. The CSF appeared as dark gray pixels in the choroidal fissure and formed the superior boundary of the hippocampal body. When the white matter of the alveus lining the ventricular surface of the hippocampus was visible, it was included in the region traced. The inferior, lateral, and medial boundaries for the hippocampal body are the same as those described above for the hippocampal head (Figs. 1 and 2).

\section{The Hippocampal Tail}

The most posterior section from which hippocampal measurements were taken was the slice on which the crus of the fornix appeared continuous and completely separate from the hippocampus and its fimbria (Figs. 1, 2C, and 3, $G$ and $H$ ). On some slices, the crus of the fornix appeared complete and separate from the hippocampus on one side before the other, and on these slices measurements were taken only from that side. This protocol excludes $5 \%$ to $10 \%$ of the hippocampus, and portions of the tail of the hippocampus were seen on slices posterior to the last slice used for measurements in some cases (Figs. 1, 2C, and 3, $G$ and $H$ ) (13). The tail of the hippocampus extends to the splenium of the corpus callosum in the most posterior sections.

Again, the inferior, lateral, and medial boundaries for the hippocampal body are the same as those described above for the hippocampal head (Figs. 1 and 2D).

\section{Statistical Analysis of Hippocampal Volumes}

A $t$ test $(\alpha=0.05)$ was used to establish that the right and left hippocampal volumes were not significantly different and that both sides could be analyzed together. A $\chi^{2}$ test was performed to test for age-related changes in hippocampal 
volume. Finally, linear regression fit and correlation analyses were performed on the normalized data.

\section{D Rendering}

To visualize the hippocampus in isolation from the rest of the brain, the 3D volumes of the segmented hippocampi were rendered and viewed from different angles. The rendered volumes were a reconstruction of the segmented area on each section on the set of sequential MR images. This step was undertaken to confirm visually the overall correctness of the segmentation procedure.

\section{Inter- and Intraobserver Variability}

Following an explicit protocol and having the traces verified for accuracy and consistency by a neuroradiologist (K.A.T.) minimized intraobserver variability (C.Y.H.). Interobserver variability was tested with two additional researchers (G.E.S., A.O.) who had never traced hippocampal volumes and had little if any previous knowledge of human hippocampal anatomy.

\section{Normalization of Hippocampal Volumes}

Normalization of pediatric hippocampal volumes was required to allow absolute volume comparison between various ages because the volumetric data failed the above-mentioned $\chi^{2}$ test. Four different methods were used to normalize hippocampal volumes to $1 \mathrm{y}$ of age.

Method A-head circumference. The first method, "A," used the patient head circumference measured at the time of the initial office visit. The date of this measurement was different from the date of the MRI. This difference was corrected by using the percentile data for standard pediatric head circumference for males and females as published by Nelhaus (20). Specifically, let $A_{\mathrm{o}}$ and $C_{\mathrm{o}}$ be the head circumference and age, respectively, at the time of the office visit, and let $A_{\mathrm{m}}$ be the age at the time of the MRI. If the point $\left(A_{\mathrm{o}}, C_{\mathrm{o}}\right)$ was on the $p$ percentile curve of the Nelhaus graph, then the corrected circumference $C_{\mathrm{m}}$ was the value such that $\left(A_{\mathrm{m}}, C_{\mathrm{m}}\right)$ was also on the $p$ percentile curve. The corrected hippocampal volume $V_{\mathrm{A}}$ was computed from the measured hippocampal volume $V_{\mathrm{m}}$ according to

$$
V_{A}=V_{m}\left(\mathrm{C}_{1} / \mathrm{C}_{\mathrm{m}}\right)
$$

where $C_{1}$ is the 50 th percentile value for head circumference at 1 y of age (20).

Method B-sphere volume. The second method, "B," used the head circumference to estimate the volume of the head. Specifically, the corrected volume for method "B" was computed as

$$
V_{B}=V_{m}\left(\mathrm{C}_{1}^{3} / \mathrm{C}_{\mathrm{m}}^{3}\right)
$$

where $V_{\mathrm{m}}, C_{\mathrm{m}}$, and $C_{1}$ are as previously defined.

Method $\boldsymbol{C}$-block volume. The third method, " $\mathrm{C}$," used a traced "block volume" of the brain along with the corrected circumference $C_{\mathrm{m}}$ to normalize the measured hippocampal volumes. The block volume as originally measured, $B_{\mathrm{m}}$, was defined as the volume of brain, excluding the brain stem, contained in the MR image slices in which the hippocampus was identified. This volume was defined by manually tracing the boundary of the brain in the slices, and the volume was computed by adding up the enclosed voxel volumes, similar to the method for determining the hippocampus volume previously described. The volume $B_{\mathrm{m}}$ had to be corrected for its position within the pediatric population similar to the correction used for head circumference. This procedure was necessary to insure that the "block volume" was reasonably independent of hippocampal volume. Because our data were not extensive enough to allow the fit of a proper growth curve, a linear regression curve meant to represent the tangent to the growth curve was fit through the $B_{\mathrm{m}}$ and $T_{\mathrm{m}}$ data where $T_{\mathrm{m}}$ represents the thickness used to define the block volume $B_{\mathrm{m}}$. From the regression fit for the block volume, a block volume $B_{1}$ for 1 y of age was obtained. An initial corrected number of slices, $T_{\mathrm{i}}$, for each subject was obtained where $T_{\mathrm{i}}$ is the value of thickness on the regression line corresponding to the patient's age. The corrected block volume thickness $T_{\mathrm{c}}$ was obtained from $T_{\mathrm{i}}$ according to

$$
T_{c}=T_{i}\left(\mathrm{C}_{\mathrm{m}} / \mathrm{C}_{50}\right)
$$

where $C_{50}$ is the 50th percentile head circumference at the age of the subject at the time of the MRI (20). From $T_{\mathrm{c}}$, a corrected number of slices was obtained as $S_{\mathrm{c}}=T_{\mathrm{c}} / h$, where $h=1.25$ $\mathrm{mm}$ is the thickness of each MR data slice in our study. If $S_{\mathrm{c}}$ was greater than the number of slices originally used to define $B_{\mathrm{m}}$, additional brain areas were traced in slices symmetrically located anterior and posterior about the original block volume until the total of $S_{\mathrm{c}}$ slices was obtained. The volumes from these additional slices were added to $B_{\mathrm{m}}$ to obtain the corrected block volume $B_{\mathrm{c}}$ with a thickness of $T_{\mathrm{c}}$. If $S_{\mathrm{c}}$ was less than the number of slices originally used to define $B_{\mathrm{m}}$, slices were symmetrically removed from the original block volume until $S_{\mathrm{c}}$ slices remained and the remaining slices were used to define a corrected block volume $B_{\mathrm{c}}$ with a thickness of $T_{\mathrm{c}}$. Finally, the normalized hippocampal volume was computed as

$$
V_{C}=V_{m}\left(\mathrm{~B}_{1} / \mathrm{B}_{\mathrm{c}}\right) \text {. }
$$

Method D-intracranial volume. A fourth method, "D," has been reported where normalization is based on estimation of intracranial volume $(7,21)$. In brief, the intracranial volume is modeled as a sphere where the height of the intracranial vault is the diameter $\left(D_{\mathrm{m}}\right)$ of the sphere whose volume is $S_{m}=$ $\pi D_{\mathrm{m}}{ }^{3} / 6$. The MRI slice that contained the first bilateral frontal ventricular horns was the index section for all of the measurements. The total area, $A_{\mathrm{m}}=\pi D_{\mathrm{m}}{ }^{2} / 4$, includes both brain tissue and CSF of the index section. The corrected hippocampal volume $\left(V_{\mathrm{D}}\right)$ was computed as

$$
V_{D}=V_{m}\left(S_{1} / S_{m}\right)
$$

where $S_{1}$ is the intracranial sphere volume at $1 \mathrm{y}$ of age as computed from a linear regression of sphere volume with age. The validity and reliability of this method has been described previously (22). 


\section{RESULTS}

\section{Flow Chart for Outlining the Pediatric Hippocampus}

The first goal of our study was to develop an algorithm for consistently outlining the pediatric hippocampus. The resultant flow chart is shown in Figures 1 and 2.

In the development of this flow chart, we have found that a number of criteria for measurement of pediatric hippocampal volumes are required for reliably outlining the margins of the hippocampus. These criteria are 1) knowledge of the shape of the hippocampus, 2) knowledge of the shape of the hippocampus on the previous and subsequent MR sections, 3) the identification of the white matter of the alveus lining the ventricular surface of the hippocampus, and 4) distinguishing pixels representing nearby gray matter and CSF.

The entire decision tree for segmenting the pediatric hippocampus is shown in Figure 1. The specific anatomical landmarks for delineating the superior boundary are different for the hippocampal head, body, and tail. The schema for these boundaries have been expanded in Figure 2, $A-C$. A typical delineation of a pediatric hippocampus from a patient is shown in Figure 3. We anticipate that the schema defined by the flow chart will provide the basis for the development of a semiautomated or fully automated software program for clinically relevant and rapid delineation of hippocampal volumes.

\section{Computed Hippocampal Volumes}

Using the schema described above, control patient hippocampal volume data were outlined and the volumes computed (Table 2). The right and left hippocampal volumes of our control patient group ranged from 1.83 to $3.21 \mathrm{~mL}$, with the mean left and right hippocampal volumes being $2.44 \mathrm{~mL}$ and $2.59 \mathrm{~mL}$, respectively. Combining left and right hippocampal volumes resulted in an average $5.04 \mathrm{~mL}$ volume. Statistical evaluation of left-right hippocampal volume differences revealed no significant differences $(p=0.07)$. However, four of six subjects had a right hippocampus that was $10 \%$ larger on average than the left (Table 2). Gender differences were not evaluated statistically, although female patients, who were also our youngest patients, tended to have the smallest hippocampi (Table 2).

Because there was no difference between the right and left hippocampal volumes, no discrimination was made between left and right in subsequent statistical tests. The $\chi^{2}$ test showed a significant relationship between volume and age in this group $(p<0.0001)$ (Fig. 4). This result was anticipated because the pediatric hippocampus becomes larger with increasing age. Therefore, to compare volumes between control patients of different ages, normalization of the hippocampal volume measurement is required.

\section{D Rendering}

The characteristic arched shape as well as the change in morphology from anterior to posterior was apparent in the 3D renderings (Fig. 5). The distribution of white and gray matter could also be visualized, with the white matter of the alveus particularly prominent along the superior surface of the hippocampus confirming that the segmentation protocol was properly followed. The shapes and orientations of the rendered hippocampi were anatomically concordant with images from neuroanatomic atlases and models $(13,16)$.

\section{Inter- and Intraobserver Variability}

After a single training session, the interobserver variability was measured to be $13 \%$. This is consistent with variability in adult hippocampal volumes (14\%) (23) but greater than that found in pediatric hippocampal volumes after extensive training (24). Intraobserver variability in an individual who did the tracings routinely (C.Y.H.) was a remarkably low $2.8 \%$. These intra- and interobserver variability measures further validate our hippocampal tracing methodology.

\section{Normalization of Hippocampal Volumes}

Method A normalization-head circumference. The corrected volumes, $V_{\mathrm{A}}$, had a correlation of $R=0.625$ in the linear regression fit (Fig. 6).

Method B normalization-sphere volume. The approximation yielded a rather poor fit to a linear volume-age relationship ( $R=0.166)$, with a slight increase in the hippocampal volume, $V_{\mathrm{B}}$, with increasing age (Fig. 6).

Method C normalization-block volume. The linear fit to $V_{\mathrm{C}}$ with respect to age resulted in a negative slope with a correlation coefficient of $R=0.589$ (Fig. 6).

Method D normalization-intracranial volume. As can be seen in Figure 6, the final method provided the most ageindependent fit to the data, but seemed to be the least reliable from a statistical standpoint $(R=0.022)$. This method was the easiest to calculate because it did not require an independent head circumference measurement.

Table 2. Computed hippocampal volumes from control pediatric patients

\begin{tabular}{|c|c|c|c|c|c|c|}
\hline \multirow[b]{2}{*}{ Control no. } & \multirow[b]{2}{*}{ Sex } & \multirow[b]{2}{*}{ Age (mo) } & \multicolumn{4}{|c|}{ Hippocampal volume (cc) } \\
\hline & & & Left & Right & Total & R-L diff* \\
\hline 1 & M & 42 & 2.81 & 2.78 & 5.59 & -0.03 \\
\hline 3 & $\mathrm{~F}$ & 55 & 3.21 & 3.17 & 6.38 & -0.04 \\
\hline 4 & M & 14 & 1.83 & 2.02 & 3.85 & 0.20 \\
\hline 5 & M & 60 & 2.45 & 2.60 & 5.05 & 0.15 \\
\hline 6 & $\mathrm{~F}$ & 31 & 2.01 & 2.38 & 4.39 & 0.37 \\
\hline
\end{tabular}

\footnotetext{
* The right hippocampal volume was subtracted from the left to highlight differences.
} 
Hippocampal Volume vs. Age

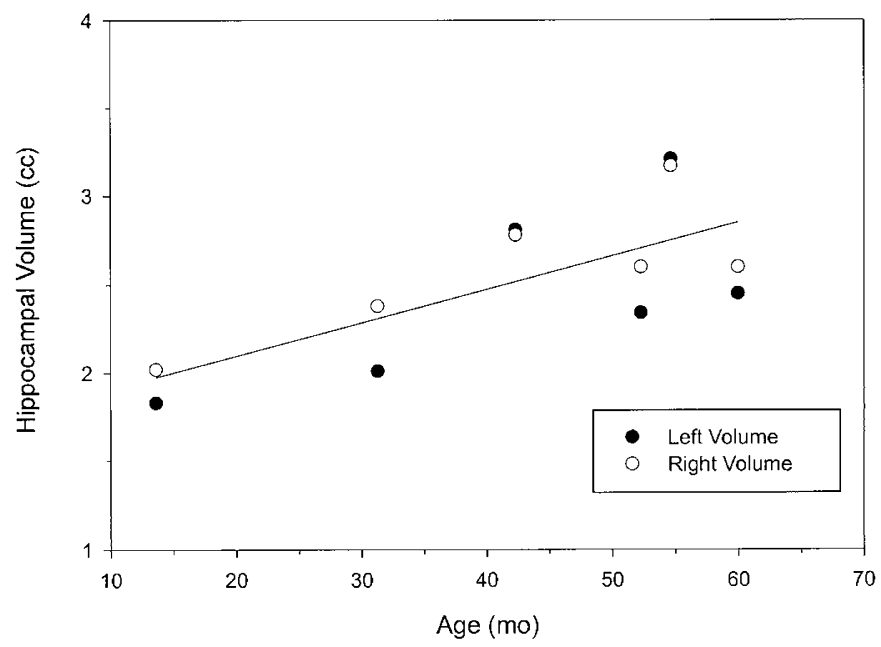

Figure 4. Hippocampal volumes of control patients. The right and left hippocampal volumes of children without temporal lobe neuropathology increase with age. Right and left hippocampal volumes are similar, with the right hippocampus slightly larger in the majority of children. A single order regression line is plotted, illustrating that the hippocampus increases with age and thus the data require normalization for accurate interindividual comparisons.

\section{DISCUSSION}

A standardized method for segmenting the pediatric hippocampus was developed based on previous descriptions for segmenting the adult hippocampus on MRI $(8,13,25)$. The flow chart was used to guide the tracing of the hippocampi of six patients without neurologic symptoms (Figs. 1 and 2). The hippocampal volumes are both age and gender dependent and larger with increasing age, and tend to be larger in males (Fig. 4). The right versus left discrepancy is consistent with previous reports $(4,8,10,13,25)$. A gender discrepancy has been reported by some groups $(8,9)$ but not others $(4,13,26)$.

$3 \mathrm{D}$ reconstruction allowed comparison of the shape of our segmented hippocampi with neuroanatomic atlases. Our hippocampi showed the forms of the hippocampal head, body, and tail from anterior to posterior, as well as the characteristic arched shape and orientation. The distributions of white and gray matter were as anticipated, with the white matter of the alveus overlying the gray matter of the hippocampus proper. Visualization of our rendered hippocampi revealed the expected shape and orientation of the hippocampus, thereby validating our method (Fig. 5).

There are few studies with which to compare pediatric hippocampal volumes. A retrospective study of pediatric hippocampal volumes reported that the normative volumes for hippocampi ranged from 1.04 to $3.47 \mathrm{~mL}$ (24). Our own results are consistent with this report. However, several other pediatric studies have reported a larger range of volumes from 2.7 to $6.55 \mathrm{~mL}(27,28)$. The large differences in the reported pediatric hippocampal volumes are likely due to differences in MRI acquisition sequences, postprocessing of the data, and methods used in delineation of the hippocampus (29). Due to this variability, we have developed a schema for outlining the
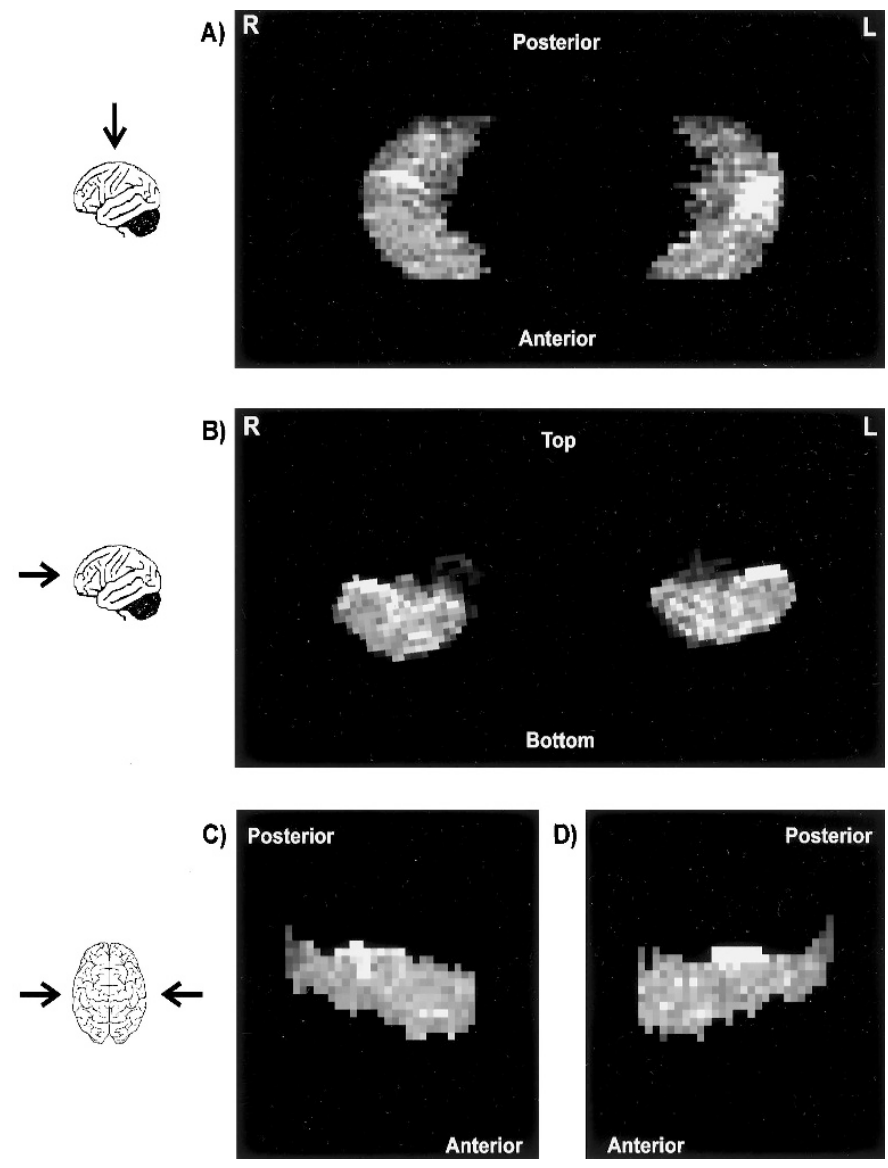

Figure 5. Rendered pediatric hippocampal volumes. The computer-rendered hippocampi shown here are the result of using the schema and algorithms detailed in Figures 1 and 2. The rendered hippocampi from superior $(A)$, anterior $(B)$, and sagittal $(C)$ views illustrate the characteristic arched shape of the hippocampus with its expected orientation. This validates that our method accurately delineates the hippocampus of children.

pediatric hippocampus that assists in standardizing hippocampal volume quantification (Figs. 1 and 2).

Unlike comparison within adult patient groups, comparison within pediatric patient groups is complicated because body growth is at a different rate than hippocampal growth. Pfluger et al. (24) described in detail hippocampal growth curves based on volume measurements. Ninety-eight percent of hippocampal growth was reached within $31 \mathrm{mo}$ in females and $86 \mathrm{mo}$ in males. A limitation of the present study was an inadequate female population that made gender differences difficult to assess.

To draw any meaningful conclusions from volumetric observations, the hippocampal volumes must be normalized, particularly if re-imaging of the patient at a later date is desired. Unfortunately, there is no available database of normal pediatric hippocampal volumes for comparison. Absolute hippocampal volumes corrected using cranial volumes have been reported to yield the greatest reduction in variance of corrected hippocampal volumes (8).

Although the weights of the patients are readily available, there is a wide variation of normal weights for young children and normalization by patient weight would not be an accurate method. Normalization using age or height are simple ap- 
Comparison of Normalization Methods

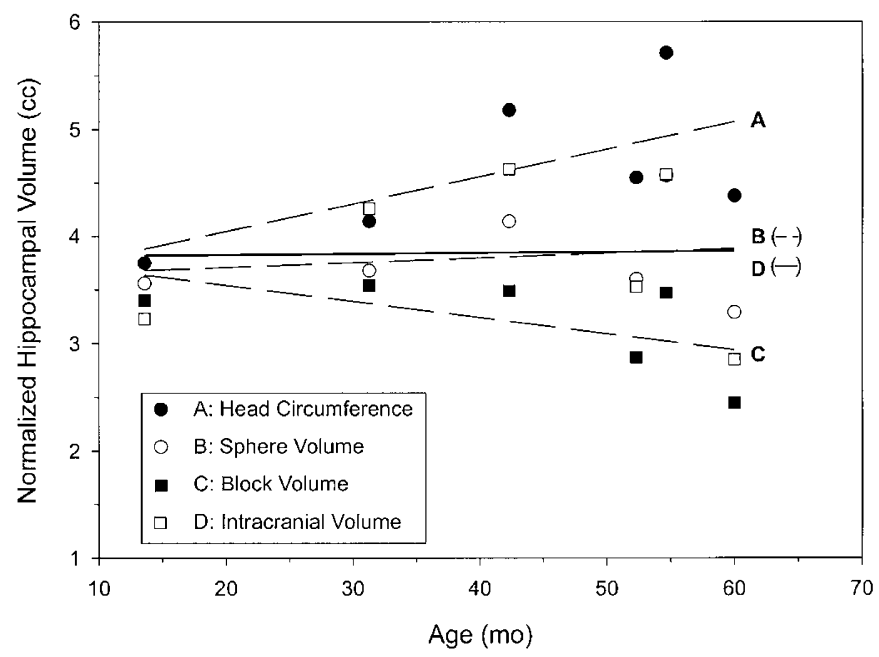

Figure 6. Comparison of normalization methods. This graph allows direct comparison of the four normalization methods (see "Results" for detailed description). A single order regression line is plotted along the best fit for each of the normalization methods. It is readily apparent that the most accurate and reliable method is estimation of intracranial volume (Method D, solid regression line, open squares). This normalization method will allow direct comparison between children and will also provide a basis for re-imaging follow-up visits.

proaches, but these are not preferable methods as the relationships of these variables with hippocampal volume do not appear to be linear and there is a wide range of heights within normal development (20).

Four different methods of normalization were evaluated in our study (Fig. 6). Adjustment for growth using head circumference or the calculation of the hippocampal block volume resulted in over- and underestimation, respectively, of the pediatric hippocampal volume. Estimation of the pediatric head volume based on a sphere calculated by head circumference yielded a good normalization. A potential weakness with these methods is that a failure to collect head circumference data would lead to insufficient information for use of as the normalization technique of choice. Furthermore, the "block volume" method also is difficult as this approach is significantly more labor intensive because it required semiautomated volume ROI selection with manual correction. We anticipated that the "block volume" method could conceivably provide the most robust normalization, but we found that this method seemed to overcorrect for the size of the hippocampus, resulting in a negative slope of the linear regression line.

The best normalization was found when estimating intracranial volume by determining the diameter of the intracranial vault from the MRI data. This last method has been described for adult hippocampal volumes in detail and provides a robust method for correcting for age and head size $(6,7,22,30)$. Our normalization results with pediatric hippocampal volume measurements are the first reported and concur with the adult studies. The intracranial volume normalization can allow direct comparisons between hippocampal volumes in children who are re-imaged. It should be noted that there are different patterns of growth for the hippocampus (right versus left) and the brain and these factors could influence corrected hippocampal volumes $(24,31)$.

An interobserver variability of $13 \%$ was observed in the quantification of hippocampal volumes, but the volumes traced by minimally trained observers were consistently lower than the volumes traced by a trained expert observer. We therefore expect that the variation in the differences of hippocampal volumes found by individual observers to be $<13 \%$. As a result, the quantification of hippocampal volumes using our method is expected to be far more sensitive to the detection of hippocampal volume changes than visual inspection of MR images.

Hippocampal segmentation is not presently practical in a clinical setting. Although accurate and reliable automatic segmentation of adult hippocampi is possible, software for this method is not widely available. Manual and semiautomatic methods are also expensive, as they are both labor and time intensive and require a single operator with good knowledge of hippocampal and surrounding anatomy. The contrast available on MR images is constantly improving as stronger magnets and new imaging techniques are developed. Improved resolution will make both automatic and semiautomatic segmentation faster and easier. As more centers develop automatic segmentation software, and as the software is commercialized, the tools should become more widely available. Although the complex anatomy of the hippocampus makes it difficult to segment, the sensitive nature of the hippocampus to seizures makes it worthwhile to study. For now, hippocampal volumetry remains a research tool, but as such it can provide valuable information about the progression of mesial temporal sclerosis and its relationship with epilepsy, both in adults with a history of a prolonged childhood seizures and in children who have just experienced such seizures.

\section{REFERENCES}

1. Sitoh Y-Y, Tien R 1998 Neuroimaging in epilepsy. J Magn Reson Imaging 8:277-288

2. Jackson GD, Berkovic SF, Duncan JS, Connelley A 1993 Optimizing the diagnosis of hippocampal sclerosis using MR imaging. AJNR Am J Neuroradiol 14:753-762

3. Jackson GD, 1994 New techniques in magnetic resonance and epilepsy. Epilepsia 35:S2-S13

4. Lee N, Tien RD, Lewis DV, Friedman AH, Felsberg GJ, Crain B, Hulette C, Osumi AK, Smith JS, VanLandingham KE, Radtke RA 1995 Fast spin-echo, magnetic resonance imaging-measured hippocampal volume: correlation with neuronal density in anterior temporal lobectomy patients. Epilepsia 36:899-904

5. Van Paesschen W, Duncan JS, Stevens JM, Connelley A 1998 Longitudinal quantitative hippocampal magnetic resonance imaging study of adults with newly diagnosed partial seizures: one-year follow-up results. Epilepsia 39:633-639

6. Briellmann RS, Jackson GD, Kalnins R, Berkovic SF 1998 Hemicranial volume deficits in patients with temporal lobe epilepsy with and without hippocampal sclerosis. Epilepsia 39:1174-1181

7. Marsh L, Morrell MJ, Shear PK, Sullivan EV, Freeman H, Marie A, Lim KO, Pfefferbaum A 1997 Cortical and hippocampal volume deficits in temporal lobe epilepsy. Epilepsia 38:576-587

8. Free SL, Bergin PS, Fish DR, Cook MJ, Shorvon SD, Stevens JM 1995 Methods for normalization of hippocampal volumes measured with MR. AJNR Am J Neuroradiol 16:637-643

9. Bhatia S, Bookheimer SY, Gaillard WD, Theodore WH 1993 Measurement of whole temporal lobe and hippocampus for MR volumetry: normative data. Neurology 43:2006-2010

10. Chee MWL, Low S, Tan JSP, Lim W, Wong J 1997 Hippocampal volumetry with magnetic resonance imaging: a cost-effective validated solution. Epilepsia 38:461465

11. Cook MJ, 1994 Mesial temporal sclerosis and volumetric investigations. Acta Neurol Scand Suppl 152:109-114

12. VanLandingham KE, Heinz ER, Cavazos JE, Lewis DV 1998 Magnetic resonance imaging evidence of hippocampal injury after prolonged focal febrile convulsions. Ann Neurol 43:413-426 
13. Watson C, Andermann F, Gloor P, Jones-Gotman M, Peters T, Evans A, Olivier A, Melanson D, Leroux G 1992 Anatomic basis of amygdaloid and hippocampal volume measurements by magnetic resonance imaging. Neurology 42:1742-1750

14. Wieshmann UC, Woermann FG, Lemieux L, Free SL, Bartlett PA, Smith SJM, Duncan JS, Stevens JM, Shorvon SD 1997 Development of hippocampal atrophy: serial magnetic resonance imaging study in a patient who developed epilepsy after generalized status epilepticus. Epilepsia 38:1238-1241

15. Duvernoy HM 1998 The Human Hippocampus-Functional Anatomy, Vascularization and Serial Sections with MRI. Springer-Verlag, Berlin, pp 5-9

16. Duvernoy HM 1998 The Human Hippocampus-Functional Anatomy, Vascularization and Serial Sections with MRI. Springer-Verlag, Berlin, pp 39-45

17. Mark LP, Daniels DL, Naidich TP, Williams AL 1993 Hippocampal anatomy and pathologic alterations on conventional MR images. AJNR Am J Neuroradiol 14:1237-1240

18. Hayman LA, Fuller GN, Cavazos JE, Pfleger MJ, Meyers CA, Jackson EF 1998 The hippocampus: normal anatomy and pathology. AJR Am J Roentgenol 171:1139-1146

19. Witter MP, Wouterlood FG, Naber PA, Van Haeften T 2000 Anatomical organization of the parahippocampal-hippocampal network. Ann NY Acad Sci 911:1-24

20. Nelhaus G, 1968 Head circumference from birth to eighteen years. Pediatrics 41:106-114

21. Sullivan EV, Marsh L, Mathalon DH, Lim KO, Pfefferbaum A 1995 Anterior hippocampal volume deficits in nonamnesic, aging chronic alcoholics. Alcohol Clin Exp Res 19:110-122

22. Mathalon DH, Sullivan EV, Rawles JM, Pfefferbaum A 1993 Correction for head size in brain-imaging measurements. Psychiatry Res Neuroimaging 50:121-139
23. Jack CR, Sharbrough FW, Twomey CK, Cascino GD, Hirschorn KA, Marsh WR, Zinsmeister AR, Scheithauer B 1990 Temporal lobe seizures: lateralization with MR volume measurements of the hippocampal formation. Radiology 175:423-429

24. Pfluger T, Weil S, Weis S, Vollmar C, Heiss D, Egger J, Scheck R, Hahn K 1999 Normative volumetric data of the developing hippocampus in children based on magnetic resonance imaging. Epilepsia 40:414-423

25. Jack Jr CR 1994 MRI-based hippocampal volume measurements in epilepsy. Epilepsia 35:S21-S29

26. Cook MJ, Fish DR, Shorvon SD, Straughan K, Stevens JM 1992 Hippocampal volumetric and morphometric studies in frontal and temporal lobe epilepsy. Brain 115:1001-1015

27. Giedd J, Snell J, Lange N, Rajapakse J, Casey B, Kozuch P, Vaituzis A, Vauss Y, Hamburger S, Kaysen D, Rapoport J 1996 Quantitative magnetic resonance imaging of human brain development: ages 4-18. Cereb Cortex 6:551-560

28. Szabo CA, Wyllie E, Siavalas EL, Najm I, Ruggieri P, Kotagal P, Luders H 1999 Hippocampal volumtery in children 6 years or younger: assessment of children with and without complex febrile seizures. Epilepsy Res 33:1-9

29. Jack Jr CR, Theodore WH, Cook M, McCarthy G 1995 MRI-based hippocampa volumetrics: data acquisition, normal ranges, and optimal protocol. Mag Reson Imaging 13:1057-1064

30. Sullivan EV, Marsh L, Mathalon DH, Lim KO, Pfefferbaum A 1995 Age-related decline in MRI volumes of temporal lobe gray matter but not hippocampus. Neurobiol Aging 16:591-606

31. Kretschmann HJ, Kammradt G, Krauthausen I, Sauer B, Wingert F 1999 Growth of the hippocampal formation in man. Bibl Anat 28:27-52 